\title{
POSITIVE-ENTROPY HAMILTONIAN SYSTEMS ON NILMANIFOLDS VIA SCATTERING
}

\author{
LEO T. BUTLER
}

\begin{abstract}
Let $\Sigma$ be a compact quotient of $T_{4}$, the Lie group of $4 \times 4$ upper triangular matrices with unity along the diagonal. The Lie algebra $\mathfrak{t}_{4}$ of $T_{4}$ has the standard basis $\left\{X_{i j}\right\}$ of matrices with 0 everywhere but in the $(i, j)$ entry, which is unity. Let $g$ be the Carnot metric, a sub-riemannian metric, on $T_{4}$ for which $X_{i, i+1},(i=1,2,3)$, is an orthonormal basis. Montgomery, Shapiro and Stolin showed that the geodesic flow of $g$ is algebraically non-integrable.

This note proves that the geodesic flow of that Carnot metric on $T \Sigma$ has positive topological entropy and is real-analytically non-integrable. It extends earlier work by Butler and Gelfreich.
\end{abstract}

\section{INTRODUCTION}

Let $G$ be a connected nilpotent Lie group with discrete subgroup $D$ and let $\Sigma=G / D$ be the corresponding homogeneous space. Each homogeneous (sub)riemannian metric on $G$ induces a locally-homogeneous metric on $\Sigma$. These leftinvariant geometries are interesting both geometrically and dynamically. A basic question is

Question 1.1. Which left-invariant geodesic flows on a compact nilmanifold have zero topological entropy?

Let $T_{n}$ be the nilpotent group of upper triangular $n \times n$ real matrices with unity on the diagonal. Montgomery, Shapiro and Stolin [6] investigate the geodesic flow of a Carnot metric on $T_{4}$; they show that it reduces to the Yang-Mills hamiltonian flow which is known to be algebraically non-integrable [9, 10. In [2, metrics on compact quotients of the 3 -step nilpotent Lie group $T_{4} \oplus T_{3}$ are constructed whose geodesic flows have positive topological entropy. In [3], Butler \& Gelfreich showed that there are riemannian and sub-riemannian metrics on $T_{4}$ which have positive topological entropy. Numerical analysis in that paper suggested that the Carnot metric of Montgomery, Shapiro and Stolin has a horseshoe, hence positive topological entropy, and is analytically non-integrable. This note proves those numerical results are, in fact, correct. In that paper, a Melnikov integral is expressed in terms of scattering data for a second-order scalar differential equation; in the present paper, this scattering data is explicitly computed in terms of $\Gamma$-functions.

The Lie algebra of $T_{4}, \mathfrak{t}_{4}$, has the standard basis consisting of those $4 \times 4$ matrices $X_{i j}$ with a unit in the $i$-th row and $j$-th column, $i<j$, and zeros everywhere else. We will restrict attention to those structures $\langle\cdot, \cdot\rangle$ where $\left\langle X_{i j}, X_{k l}\right\rangle=b_{i j}$ when $i=k, j=l$ and zero otherwise. The standard riemannian metric has $b_{i j}=1$ for all $i, j$; the standard Carnot sub-riemannian metric studied in [6] has $b_{12}=b_{23}=$ $b_{34}=1$ and all other coefficients zero.

Date: March 2, 2022.

2010 Mathematics Subject Classification. 37J30; 53C17, 53C30, 53D25.

Key words and phrases. Sub-riemannian geometry; nilmanifold; topological entropy; geodesic flows.

The author thanks Adri Olde Daalhuis for his helpful comments on an early draft of this paper. 
Theorem 1.1. Let $\Sigma$ be a homogeneous space of $T_{4}$. The topological entropy of the geodesic flows of the standard riemannian and Carnot metrics is positive.

This theorem is proven by reducing the flows to hamiltonian flows on $\mathfrak{t}_{4}^{*}$, the dual of the Lie algebra $\mathfrak{t}_{4}$ of $T_{4}$. The Poisson sub-algebra of left-invariant hamiltonians on $T^{*} T_{4}$ is naturally identified with the hamiltonians on $\mathfrak{t}_{4}^{*}$ with the natural Poisson structure. The Lie group's co-adjoint action is by Poisson automorphisms and a co-adjoint orbit $\mathcal{O} \subset \mathfrak{t}_{4}^{*}$ is a symplectic submanifold to which all such hamiltonian vector fields are tangent. A quadratic hamiltonian $h: \mathfrak{t}_{4}^{*} \rightarrow \mathbf{R}$ is diagonal if it is expressed as $h=\sum_{i<j} a_{i j} X_{i j}^{2}$ for some constants $a_{i j}$.

Theorem 1.2. Let $h: \mathfrak{t}_{4}^{*} \rightarrow \mathbf{R}$ be a diagonal hamiltonian with $a_{13} a_{34}=a_{12} a_{24}$ and let $E_{h}$ be the hamiltonian, or Euler, vector field of $h$ on $\mathfrak{t}_{4}^{*}$. There is an open set of regular co-adjoint orbits $\mathcal{O} \subset \mathfrak{t}_{4}^{*}$, such that $E_{h} \mid \mathcal{O}$ has a horseshoe. In particular, the Euler vector field of the standard Carnot metric has positive topological entropy and is real analytically non-integrable.

Theorem 1.2 is proven by expressing a Melnikov integral as a quadratic form in 2 -variables with coefficients that are obtained by solving a scattering problem; these coefficients are naturally expressed in terms of $\Gamma$-functions involving a parameter, called $\alpha$ below, that depends on the metric coefficients $a_{i j}$ and the co-adjoint orbit. Note that 3 asserts that the horseshoe exists on all but countably many regular co-adjoint orbits; this is inaccurate. That paper shows the horseshoe exists for all but countably many real values of the invariant $\alpha$; however, $\alpha$ may be imaginary on an open set. This is explained in figure 1 below. As noted in [3], when $a_{13}=0$, the invariant $\alpha$ is constant and one cannot therefore conclude that there is a horseshoe on any of the co-adjoint orbits. The standard Carnot metric of [6] falls into this case $(\alpha=1)$. The present paper uses an alternative approach that shows the existence of a horseshoe for all non-zero real values of $\alpha$. This is strong enough to prove the existence of a horsehoe on an open set of co-adjoint orbits, even when $a_{13}$ vanishes. It remains an open question if the Euler vector field has a horseshoe on a co-adjoint orbit where $\alpha$ is imaginary.

Theorem 1.2 implies, from the structural stability of the horseshoe, that there is an open set $W$ of quadratic hamiltonians on $\mathfrak{t}_{4}^{*}$ each of which has a horseshoe; further, the $\operatorname{Aut}\left(T_{4}\right)$ orbit of $W$ has this property, too. This motivates the following:

Question 1.2. Does there exist a quadratic hamiltonian on $\mathbf{t}_{4}^{*}$ which induces a nondegenerate (sub-)riemannian structure on $T_{4}$ and which is completely integrable or has zero topological entropy?

If one drops the non-degeneracy condition, then the answer is trivially yes to both questions, as witnessed by $h=X_{14}^{2}$, which is a Casimir.

1.1. Outline. This note is organized as follows: section 2 reviews the derivation of the Melnikov form from [3] section 3 computes the integrals that arise in the Melnikov form in terms of the scattering matrices at $\pm \infty$ in a general scattering problem; section 4 demonstrates the non-degeneracy of the Melnikov form for the particular form arising from section 2 and completes the proof of theorems 1.1 and 1.2

\section{BACKGROUND}

This section recalls a number of facts about left-invariant hamiltonian systems on the cotangent bundle of a Lie group; see also [5, 3]. 
2.1. Poisson geometry of left-invariant hamiltonians. A Poisson manifold is a smooth manifold $M$ such that $C^{\infty}(M)$ is equipped with a skew-symmetric bracket $\{$,$\} that makes \left(C^{\infty}(M),\{\},\right)$ into a Lie algebra of derivations of $C^{\infty}(M)$. The centre of $\left(C^{\infty}(M),\{\},\right)$ is the set of Casimirs. A Casimir is a first integral of all hamiltonian vector fields.

The dual space of a Lie algebra gives an example of a Poisson manifold that is not (in general) a symplectic manifold. Let $\mathfrak{g}$ be a finite-dimensional real Lie algebra and let $\mathfrak{g}^{*}$ be the dual vector space of $\mathfrak{g}$. $T_{p}^{*} \mathfrak{g}^{*}$ is identified with $\mathfrak{g}$ for all $p \in \mathfrak{g}^{*}$. The Poisson bracket on $\mathfrak{g}^{*}$ is defined for all $f, h \in C^{\infty}\left(\mathfrak{g}^{*}\right)$ and $p \in \mathfrak{g}^{*}$ by

$$
\{f, h\}(p):=-\left\langle p,\left[d f_{p}, d h_{p}\right]\right\rangle,
$$

where $\langle\cdot, \cdot\rangle: \mathfrak{g}^{*} \times \mathfrak{g} \rightarrow \mathbf{R}$ is the natural pairing. Recall that for $\xi \in \mathfrak{g}, \operatorname{ad}_{\xi}^{*}:$ $\mathfrak{g}^{*} \rightarrow \mathfrak{g}^{*}$ is the linear map defined by $\left\langle\operatorname{ad}_{\xi}^{*} p, \eta\right\rangle=-\langle p,[\xi, \eta]\rangle \cdot \operatorname{ad}^{*}: \mathfrak{g} \rightarrow g l\left(\mathfrak{g}^{*}\right)$ is the co-adjoint representation. For any $h \in C^{\infty}\left(\mathfrak{g}^{*}\right)$, the hamiltonian vector field $E_{h}=\{\bullet, h\}$ equals $-\operatorname{ad}_{d h(p)}^{*} p$.

Let $G$ be a connected Lie group whose Lie algebra is $\mathfrak{g}$. The adjoint representation of $G$ on $\mathfrak{g}, \operatorname{Ad}_{g} \xi=\left.\frac{d}{d t}\right|_{t=0} g \exp (t \xi) g^{-1}$, induces the co-adjoint representation $\left\langle\operatorname{Ad}_{g}^{*} p, \xi\right\rangle=\left\langle p, \operatorname{Ad}_{g^{-1}} \xi\right\rangle$ for all $p \in \mathfrak{g}^{*}, g \in G$ and $\xi \in \mathfrak{g}$. As each vector field $p \rightarrow \operatorname{ad}_{\xi}^{*} p$ is hamiltonian on $\mathfrak{g}^{*}$, with linear hamiltonian $h_{\xi}(p)=-\langle p, \xi\rangle$, the coadjoint action of $G$ on $\mathfrak{g}^{*}$ preserves the Poisson bracket. The orbits of the co-adjoint action are called the co-adjoint orbits. Each co-adjoint orbit is a homogeneous $G$ space, and every hamiltonian vector field on $\mathfrak{g}^{*}$ is tangent to each co-adjoint orbit. For this reason, the Poisson bracket $\{\cdot, \cdot\}_{\mathfrak{g}^{*}}$ restricts to each co-adjoint orbit, and is non-degenerate on each co-adjoint orbit. Thus, the co-adjoint orbits are naturally symplectic manifolds. A Casimir is necessarily constant on each co-adjoint orbit, and in many cases (as in this paper) each co-adjoint orbit is the common level set of all Casimirs.

The hamiltonian flow of a left-invariant hamiltonian $H$ on $T^{*} G$ has the equations of motion:

$$
X_{H}(g, p)=\left\{\begin{array}{l}
\dot{g}=T_{e} L_{g} d h(p), \\
\dot{p}=-\operatorname{ad}_{d h(p)}^{*} p,
\end{array}\right.
$$

The reduction of the vector field $X_{H}$ to $\mathfrak{g}^{*}$ is the Euler vector field $E_{h}$.

2.2. Poisson geometry of $T^{*} T_{4}$. The Lie algebra of $T_{4}$ is

$$
\mathfrak{t}_{4}=\left\{\left[\begin{array}{cccc}
0 & x & z & w \\
0 & 0 & y & u \\
0 & 0 & 0 & v \\
0 & 0 & 0 & 0
\end{array}\right]: u, v, w, x, y, z \in \mathbf{R}\right\} .
$$

Let $p_{\bullet}$ be the coordinate functions on $\mathfrak{t}_{4}^{*}$ dual to the above coordinates on $\mathfrak{t}_{4}$. The Poisson bracket on $t_{4}^{*}$ is:

$$
\begin{array}{ll}
\left\{p_{x}, p_{y}\right\}=-p_{z}, & \left\{p_{x}, p_{u}\right\}=-p_{w}, \\
\left\{p_{y}, p_{v}\right\}=-p_{u}, & \left\{p_{z}, p_{v}\right\}=-p_{w} .
\end{array}
$$

There are two independent Casimirs of $\mathfrak{t}_{4}^{*}$ are $K_{1}(p)=p_{w}, K_{2}(p)=p_{w} p_{y}-p_{z} p_{u}$. Let $K: \mathfrak{t}_{4}^{*} \rightarrow \mathbf{R}^{2}$ be defined by $K=\left(K_{1}, K_{2}\right)$. The level sets of $K$ are the co-adjoint orbits of $T_{4}$ 's action on $\mathfrak{t}_{4}^{*}$ and will be denoted by $\mathcal{O}_{k}$, where $k=\left(k_{1}, k_{2}\right)$. We will say that $\mathcal{O}_{k}$ is a regular co-adjoint orbit if $k_{1} \neq 0$.

Lemma 2.1. Each regular co-adjoint orbit $\mathcal{O}_{k}$ is symplectomorphic to $T^{*} \mathbf{R}^{2}$ equipped with its canonical symplectic structure.

Proof. Indeed, the right-hand column of the commutation relations (3) shows that when $k_{1}=p_{w} \neq 0$, the coordinates $\left(p_{x}, p_{u}, p_{z}, p_{v}\right)$ are conformally symplectic and the first column is a consequence of $K_{2}=k_{2}$ constant on $\mathcal{O}_{k}$. See [3]. 
2.3. The hamiltonians. Let $a_{i j} \geq 0$ be constants such that $a_{12} a_{23} a_{34} \neq 0$ and $a_{13} a_{34}=a_{12} a_{24}$. Define

$$
4 h(p)=a_{12} p_{x}^{2}+a_{23} p_{y}^{2}+a_{13} p_{z}^{2}+a_{24} p_{u}^{2}+a_{34} p_{v}^{2}+a_{14} p_{w}^{2}
$$

As shown in [3], there is a change of coordinates that transforms $h \mid \mathcal{O}_{k}$ to

$$
2 \mathbf{h}_{k}=\left(x^{2}-\xi X^{2}+\nu X^{4}\right)+\left(y^{2}+\omega Y^{2}+\nu Y^{4}-2 \nu X^{2} Y^{2}\right)
$$

where $(x, X, y, Y)$ are canonically conjugate coordinates, $\xi=-\left(a_{13} a_{34} k_{1}^{2}+a_{23} k_{2} \sqrt{a_{12} a_{34}}\right)$, $\omega=\xi+2 a_{13} a_{34} k_{1}^{2}=a_{13} a_{34} k_{1}^{2}-a_{23} k_{2} \sqrt{a_{12} a_{34}}$.

The Casimirs may be rescaled to $c_{1}=\sqrt{a_{13} a_{34}} k_{1}$ and $c_{2}=a_{23} k_{2} \sqrt{a_{12} a_{34}}$. In this case, $\xi=-c_{1}^{2}-c_{2}$ and $\omega=c_{1}^{2}-c_{2}$. The ratio $\frac{\omega}{\xi}$ is negative when $\xi<0<\omega$, that is, when $c_{1}^{2}>c_{2}>-c_{1}^{2}$. Otherwise, either $\xi<\omega<0$ or $0<\xi<\omega$. If $e>0$, the energy level $\left\{\mathbf{h}_{k}=e\right\}$ intersects the set $\mathrm{S}$ of Casimir values where the ratio $\frac{\omega}{\xi}>0$, i.e. where the origin is a saddle-centre for $\mathbf{h}_{k}$ (figure 1). In the degenerate case where $a_{13}=0, c_{1} \equiv 0$ and the ratio $\omega / \xi \equiv 1$, except when $c_{2}=0$, where it is undefined.

FiguRE 1. The regions in the space of rescaled Casimir values where (5) has saddle-centre (S) and centre-centre (C) equilibria at the origin.

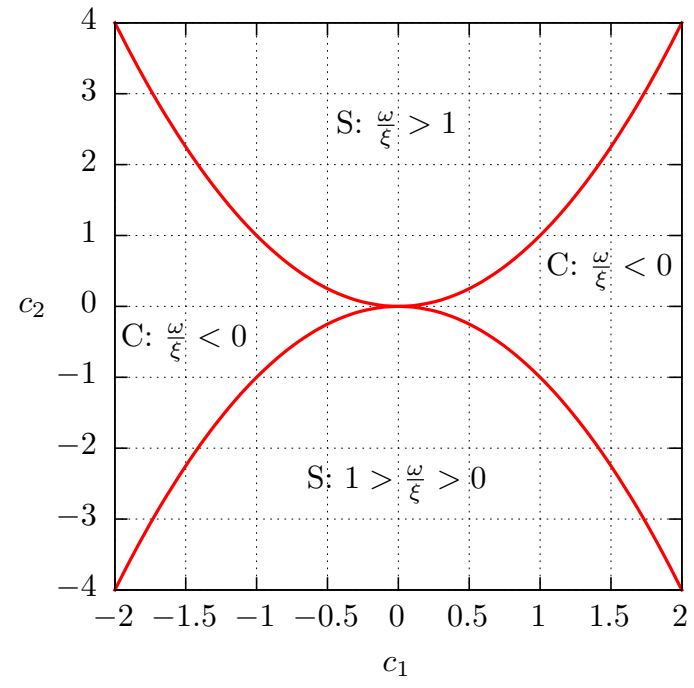

2.3.1. The geodesic flow and Euler equations. When $\frac{\omega}{\xi}>0$, there is a second change of variables that transforms $\mathbf{h}_{k}$ into a constant multiple of

$$
2 \mathbf{h}=x^{2}+\left(X^{2}-\frac{1}{2}\right)^{2}+y^{2}+\alpha^{2} Y^{2}+Y^{4}-2 X^{2} Y^{2}
$$

For all $\epsilon>0$, the rescaling $(y, Y) \mapsto(y, Y) / \sqrt{\epsilon}$ transforms the hamiltonian vector-field of $\mathbf{h}$ (equation 6) to the non-hamiltonian vector-field

$$
\mathcal{X}_{\epsilon}= \begin{cases}\dot{X}=x, & \dot{Y}=y, \\ \dot{x}=X-2 X^{3}+2 \epsilon X Y^{2}, & \dot{y}=\left[-\alpha^{2}+2 X^{2}\right] Y-2 \epsilon Y^{3} .\end{cases}
$$

2.3.2. The normally hyperbolic manifold $S$. The plane

$$
S=\{(x, X, y, Y): x=X=0\}
$$

is $\mathcal{X}_{\epsilon}$-invariant for all $\epsilon$. As shown in [3], $S$ is normally-hyperbolic for all $\epsilon$. 
2.3.3. The stable and unstable manifolds of $S$. The function $h: T^{*} \mathbf{R}^{2} \rightarrow \mathbf{R}, h=$ $x^{2}+\left(X^{2}-\frac{1}{2}\right)^{2}$ is a first integral of $\mathcal{X}_{0}$. The set $h^{-1}\left(\frac{1}{4}\right)$ is the stable and unstable manifold of $S$, which we denote by $W_{0}^{ \pm}(S)$. On $W_{0}^{ \pm}(S)-S$, the flow of $\mathcal{X}_{0}$ satisfies

$$
\begin{array}{ll}
X= \pm \operatorname{sech}\left(t+t_{0}\right), & x=\mp \tanh \left(t+t_{0}\right) \operatorname{sech}\left(t+t_{0}\right), \\
Y=c_{0} Y_{0}\left(t+t_{0}\right)+c_{1} Y_{1}\left(t+t_{0}\right), & y=\dot{Y},
\end{array}
$$

where $X(0)= \pm \operatorname{sech}\left(t_{0}\right), x(0)=\mp \tanh \left(t_{0}\right) \operatorname{sech}\left(t_{0}\right)^{2}$ and $\left\{Y_{j}\right\}$ is a basis of solutions to the initial-value problem

$$
\begin{aligned}
& \ddot{Y}+\left[\alpha^{2}-2 \operatorname{sech}(t)^{2}\right] Y=0, \quad \text { such that } Y(0)=Y_{0, j}, \dot{Y}(0)=\dot{Y}_{0, j} \\
& \text { and } W=Y_{0,0} \dot{Y}_{0,1}-Y_{0,1} \dot{Y}_{0,0} \neq 0
\end{aligned}
$$

while $Y(0)=c_{0} Y_{0}\left(t_{0}\right)+c_{1} Y_{1}\left(t_{0}\right), y(0)=c_{0} \dot{Y}_{0}\left(t_{0}\right)+c_{1} \dot{Y}_{1}\left(t_{0}\right)$. The particular choice of basis is discussed in section 4 .

Given a basis of solutions, this determines a coordinate system $\left(t_{0}, c_{0}, c_{1}\right)$ on the stable and unstable manifolds $W_{0}^{ \pm}(S)$ of $S$ for $\mathcal{X}_{0}$.

2.3.4. The Melnikov function. By well-known results [4, the perturbed stable and unstable manifolds of $S, W_{\epsilon}^{ \pm}(S)$ for $\mathcal{X}_{\epsilon}$ are, on compact sets, graphs over $W_{0}^{ \pm}(W)$. The Melnikov function $m$ measures the $O(\epsilon)$ separation of these graphs. In this case, it is a quadratic form in the coordinates $c_{0}, c_{1}[3]$ :

$$
\begin{aligned}
m\left(t_{0}, c_{0}, c_{1}\right) & =m_{00} c_{0}^{2}+2 m_{01} c_{0} c_{1}+m_{11} c_{1}^{2} & & \text { where } \\
m_{i j} & =\int_{\tau \in \mathbf{R}} \dot{q}(\tau) Y_{i}(\tau) Y_{j}(\tau) \mathrm{d} \tau, & & \text { and } q(\tau)=2 \operatorname{sech}(\tau)^{2} .
\end{aligned}
$$

\section{IMPROPER INTEGRALS VIA SCATTERING}

In [3], the coefficients $m_{i j}$ are computed in terms of the asymptotic phase angle between an even and odd solution to (9). This section examines an alternative route to computing the coefficients $m_{i j}$ for a general class of scattering problems and integrals like those in 111 .

Definition 3.1. Two functions $f, g \in C^{1}(\mathbf{R})$ are said to be asymptotically equal at $+\infty$, written $f \widetilde{+}$, if, for each $\epsilon>0$, there is a $T>0$ such that

$$
t \geq T \quad \Longrightarrow \quad|f(t)-g(t)|+\left|f^{\prime}(t)-g^{\prime}(t)\right|<\epsilon .
$$

The definition of asymptotic equality at $-\infty$ is similar and denoted by $\simeq$.

Let $q \in C^{1}(\mathbf{R}) \cap L^{1}(\mathbf{R})$ and $\alpha>0$. Since $q \widetilde{ \pm} 0$, there are solutions $w_{j}^{ \pm}$, $j \in\{0,1\}$, to the differential equation

$$
\ddot{w}+\left[\alpha^{2}-q(t)\right] w=0
$$

such that

$$
w_{j}^{ \pm}(t) \simeq \exp \left((-1)^{j} i \alpha t\right) .
$$

Given two solutions $w_{0}, w_{1}$ to $(13)$-which are not necessarily the solutions (14)-, let

$$
J_{ \pm}=\lim _{ \pm t \rightarrow \infty} \dot{w}_{0}(t) \dot{w}_{1}(t)+\alpha^{2} w_{0}(t) w_{1}(t) .
$$

Since, for each $\sigma \in\{ \pm\},\left\{w_{0}^{\sigma}, w_{1}^{\sigma}\right\}$ is a basis of the solution space to 13 , there are constants $a_{i j}^{\sigma}$ such that

$$
\left[\begin{array}{l}
w_{0} \\
w_{1}
\end{array}\right]=\left[\begin{array}{ll}
a_{00}^{\sigma} & a_{01}^{\sigma} \\
a_{10}^{\sigma} & a_{11}^{\sigma}
\end{array}\right]\left[\begin{array}{l}
w_{0}^{\sigma} \\
w_{1}^{\sigma}
\end{array}\right] .
$$

Lemma 3.1. The limits 15 exist and are equal to

$$
J_{\sigma}=2 \alpha^{2}\left[a_{01}^{\sigma} a_{10}^{\sigma}+a_{00}^{\sigma} a_{11}^{\sigma}\right], \quad \text { for } \sigma \in\{ \pm\}
$$


Proof. Let $\sigma$ be + or - . One sees that

$$
\begin{aligned}
& \dot{w}_{0} \dot{w}_{1}+\alpha^{2} w_{0} w_{1} \\
= & \left(a_{00}^{\sigma} \dot{w}_{0}^{\sigma}+a_{01}^{\sigma} \dot{w}_{1}^{\sigma}\right)\left(a_{10}^{\sigma} \dot{w}_{0}^{\sigma}+a_{11}^{\sigma} \dot{w}_{1}^{\sigma}\right)+\alpha^{2}\left(a_{00}^{\sigma} w_{0}^{\sigma}+a_{01}^{\sigma} w_{1}^{\sigma}\right)\left(a_{10}^{\sigma} w_{0}^{\sigma}+a_{11}^{\sigma} w_{1}^{\sigma}\right) \\
\widetilde{\sigma}- & \alpha^{2}\left(a_{00}^{\sigma} \exp (i \alpha t)-a_{01}^{\sigma} \exp (-i \alpha t)\right)\left(a_{10}^{\sigma} \exp (i \alpha t)-a_{11}^{\sigma} \exp (-i \alpha t)\right) \\
& +\alpha^{2}\left(a_{00}^{\sigma} \exp (i \alpha t)+a_{01}^{\sigma} \exp (-i \alpha t)\right)\left(a_{10}^{\sigma} \exp (i \alpha t)+a_{11}^{\sigma} \exp (-i \alpha t)\right)
\end{aligned}
$$

which implies (17).

Theorem 3.1. Let $w_{0}, w_{1}$ be solutions to $(13)$. The integral

$$
I=\int_{-\infty}^{\infty} \dot{q}(t) w_{0}(t) w_{1}(t) \mathrm{d} t
$$

exists and equals $J_{-}-J_{+}$, that is,

$$
I=2 \alpha^{2}\left[a_{01}^{-} a_{10}^{-}+a_{00}^{-} a_{11}^{-}-a_{01}^{+} a_{10}^{+}-a_{00}^{+} a_{11}^{+}\right] .
$$

Proof. The proof is similar to that in [3]. Let $I_{\sigma}=\sigma \int_{0}^{\sigma \infty} \dot{q}(t) w_{0}(t) w_{1}(t) \mathrm{d} t$ for $\sigma \in\{ \pm\}$, so that $I=I_{+}+I_{-}$. Integration by parts shows that $I_{+}=-J_{+}+C$ and $I_{-}=J_{-}-C$ where $C=\left[\alpha^{2}+q(0)\right] w_{0}(0) w_{1}(0)+\dot{w}_{0}(0) \dot{w}_{1}(0)$ is a boundary datum.

The following is useful in computing the Melnikov coefficients $m_{i j}$ (11).

Corollary 3.1. Let $I_{i j}$ denote the integral 18 with $w_{0}=w_{i}^{-}$and $w_{1}=w_{j}^{-}$for $i, j \in\{0,1\}$. Then

$$
I_{i i}=-4 \alpha^{2} a_{i 0}^{+} a_{i 1}^{+} \quad I_{01}=I_{10}=2 \alpha^{2}\left(1-a_{00}^{+} a_{11}^{+}-a_{01}^{+} a_{10}^{+}\right)
$$

where $\left[a_{i j}^{+}\right]$is the connection matrix (16). Moreover

$$
\operatorname{det}\left[I_{i j}\right]=-4 \alpha^{4}\left(\left(1-a_{00}^{+} a_{11}^{+}-a_{01}^{+} a_{10}^{+}\right)^{2}-4 a_{00}^{+} a_{11}^{+} a_{01}^{+} a_{10}^{+}\right) .
$$

\section{THE SCATTERING COEFFICIENTS AND SPLITTING OF THE INVARIANT MANIFOLDS}

To compute the $m_{i j}$ in (11), it is useful to transform the differential equation (9) into a form that reveals its solubility in terms of hypergeometric functions. Substitution of $z=\tanh (t)$ transforms the differential equation $(9)$ into the Legendre differential equation [8, p. 324]

$$
\left(1-z^{2}\right) Y^{\prime \prime}-2 z Y^{\prime}+\left(\nu(\nu+1)-\frac{\mu^{2}}{1-z^{2}}\right) Y=0
$$

where $\mu=i \alpha, \nu=-\frac{1}{2}+\frac{\sqrt{-7}}{2}$ and ${ }^{\prime}=\frac{\mathrm{d}}{\mathrm{d} z}$.

Let $F(a, b ; c ; z)$ be the $(2,1)$ hypergeometric function with moduli $a, b, c \in \mathbf{C}$ and argument $z \in \mathbf{C}$ [8, p. 281], [7, \$15.2.1]. There are four privileged solutions to (22) that are expressed in terms of these hypergeometric functions, namely,

$$
\begin{array}{ll}
Y_{0}^{+}=\left[\frac{1+z}{1-z}\right]^{\frac{i \alpha}{2}} F\left(a, b ; c ; \frac{1-z}{2}\right) & Y_{1}^{+}=\left[\frac{1+z}{1-z}\right]^{-\frac{i \alpha}{2}} F\left(a, b ; \bar{c} ; \frac{1-z}{2}\right) \\
Y_{0}^{-}=\left[\frac{1-z}{1+z}\right]^{\frac{i \alpha}{2}} F\left(a, b ; c ; \frac{1+z}{2}\right) & Y_{1}^{-}=\left[\frac{1-z}{1+z}\right]^{-\frac{i \alpha}{2}} F\left(a, b ; \bar{c} ; \frac{1+z}{2}\right),
\end{array}
$$

where $a=\frac{1}{2}+\frac{\sqrt{-7}}{2}, b=\bar{a}$ and $c=1-i \alpha$ [8, p. 286]. (The notation is explained thus: the group $\mathbf{Z}_{2} \oplus \mathbf{Z}_{2}$ acts by automorphisms of 22 by changing the sign of $\alpha$ 
and $z$ independently.) From the fact that as $\pm t \rightarrow \infty, \pm z \rightarrow 1$ and $F(a, b ; c ; 0)=1$, it is apparent that

$$
\begin{array}{ll}
Y_{0}^{+} \simeq \exp (i \alpha t) & Y_{1}^{+} \simeq \exp (-i \alpha t) \\
Y_{0}^{-} \simeq \exp (i \alpha t) & Y_{1}^{-} \simeq \exp (-i \alpha t),
\end{array}
$$

viewed as functions of $t=\tanh ^{-1}(z)$.

The linear transformation rules for hypergeometric functions [1, 15.3.3, 15.3.6] imply the relations

$$
\begin{aligned}
& Y_{0}^{-}=A Y_{0}^{+}+B Y_{1}^{+}, \quad Y_{1}^{-}=\bar{B} Y_{0}^{+}+\bar{A} Y_{1}^{+} \quad \text { where } \\
& A=\frac{\Gamma(c) \Gamma(1-c)}{\Gamma(a) \Gamma(b)} \quad B=\frac{\Gamma(c) \Gamma(c-1)}{\Gamma(c-a) \Gamma(c-b)}
\end{aligned}
$$

so the connection matrices are

$$
\left[a_{i j}^{-}\right]=\left[\begin{array}{ll}
1 & 0 \\
0 & 1
\end{array}\right] \quad\left[a_{i j}^{+}\right]=\left[\begin{array}{cc}
A & B \\
\bar{B} & \bar{A}
\end{array}\right] .
$$

Lemma 4.1. Let $s=\sqrt{7} / 2$. Then

$$
|B|^{2}=\frac{\cosh (2 \pi \alpha)+\cosh (2 \pi s)}{\cosh (2 \pi \alpha)-1}, \quad|A|^{2}=\left(\frac{\cosh (\pi s)}{\sinh (\pi \alpha)}\right)^{2} \text {. }
$$

So, $|B|$ exceeds unity for all $\alpha \in \mathbf{R}, \alpha \neq 0$ and $|A| /|B|$ is maximized at $\alpha=0$ and decreases monotonically to 0 as $\alpha \rightarrow \infty$.

Proof. Assume that $x \neq 0$. The reflection formula for the $\Gamma$-function implies that $\left|\Gamma\left(\frac{1}{2}+i x\right)\right|^{2}=\frac{\pi}{\cosh (\pi x)},|\Gamma(i x)|^{2}=\frac{\pi}{|x \sinh (\pi x)|}$ and $|\Gamma(1+i x)|^{2}=\frac{\pi|x|}{|\sinh (\pi x)|}$, ๆ 6.1.17, 6.1.29-31]. Then, since $c=1-i \alpha$ and $c-a=\frac{1}{2}+i(-\alpha-s)$,

$$
\begin{aligned}
|B|^{2} & =\left|\frac{\Gamma(1-i \alpha) \Gamma(-i \alpha)}{\Gamma\left(\frac{1}{2}+i(-\alpha-s)\right) \Gamma\left(\frac{1}{2}+i(-\alpha+s)\right)}\right|^{2} \\
& =\frac{\cosh (\pi(-\alpha-s)) \cosh (\pi(-\alpha+s))}{\sinh (\pi \alpha)^{2}}
\end{aligned}
$$

which yields the first part of (27) and implies $|B| \geq 1$ and $>1$ if $\alpha \neq 0$. A similar computation shows the second part. This implies that

$$
|A|^{2} /|B|^{2}=\cosh (\pi s)^{2} /\left(\cosh (\pi s)^{2}+\cosh (\pi \alpha)^{2}-1\right) \leq 1
$$

and $<1$ when $\alpha \neq 0$ and decreases monotonically as $\alpha \rightarrow \infty$.

Theorem 4.1. The Melnikov form with coefficients $m_{i j}$ (11) for the basis $Y_{j}=Y_{j}^{-}$ is non-degenerate and indefinite for all $\alpha \in \mathbf{R}, \alpha \neq 0$.

Proof. When corollary 3.1 is applied, with the connection coefficients in (25), one computes that $m_{i j}=I_{i j}$ so

$$
\operatorname{det}\left[m_{i j}\right]=-4 \alpha^{4}\left(\left(1-|A|^{2}-|B|^{2}\right)^{2}-4|A|^{2}|B|^{2}\right) .
$$

By hypothesis, $\alpha \neq 0$, so $\operatorname{det}\left[m_{i j}\right]$ vanishes iff $|A| \pm|B|= \pm 1$. By lemma 4.1, the only possible equation to be satisfied is $|A|=|B|-1$. If this latter equation is satisfied, then

$$
[\cosh (\pi s)+\sinh (\pi \alpha)]^{2}=\cosh (\pi s)^{2}+\cosh (\pi \alpha)^{2}-1
$$

which implies that $\alpha=0$. Therefore, $\operatorname{det}\left[m_{i j}\right] \neq 0$ for all real, non-zero $\alpha$.

Indefiniteness of the Melnikov form follows from the even-ness of the potential $q(t)=-2 \operatorname{sech}(t)^{2}$ : non-trivial even and odd solutions to 9 exist and the Melnikov form vanishes on these solutions by Theorem 3.1 . 


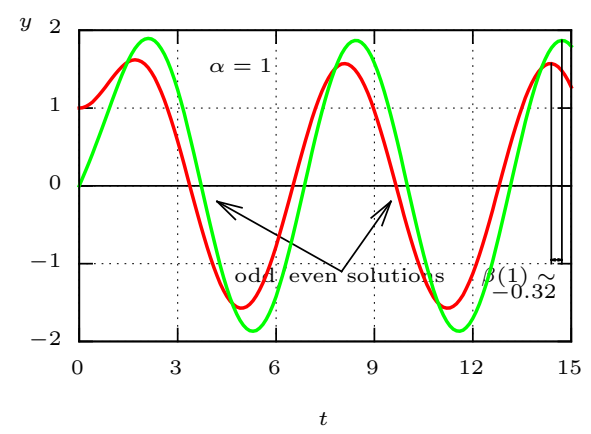

(a)

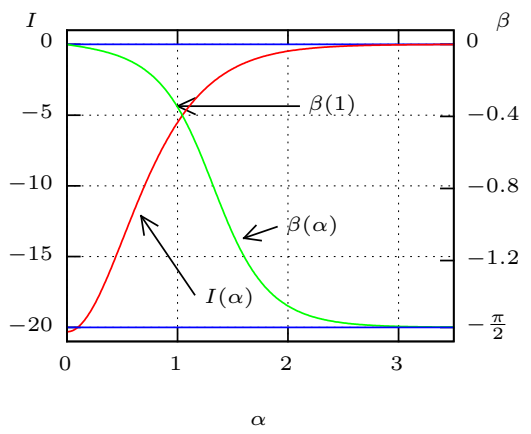

(b)

Figure 2. 2(a) Fundamental solutions to the differential equations $(9)$ with $\alpha=1$. 2(b), Left axis: the integral $I(19)$ as a function of $\alpha$ with the solutions in figure 2(a) 2(b) Right axis: the asymptotic phase angle $\beta$ between even \& odd solutions $Y_{0} \& Y_{1}$ of $(9)$.

Remark 1. Let us compare the results for the Melnikov form (11) to that obtained in [3]. The Melnikov form $\left[m_{i j}\right]$ relative to the basis $\left\{Y_{0}^{+}, Y_{1}^{+}\right\}$has been computed to be

$$
M_{y}=-2 \alpha^{2}\left[\begin{array}{cc}
2 A B & |B|^{2}+|A|^{2}-1 \\
|B|^{2}+|A|^{2}-1 & 2 \bar{A} \bar{B}
\end{array}\right] .
$$

Let $W_{0}, W_{1}$ be a pair of solutions whose Wronskian matrix is the identity at $z=0$; in particular, $W_{0}$ (resp. $W_{1}$ ) is an even (resp. odd) solution. The Melnikov form in this basis is equal to

$$
M_{w}=\underbrace{2 \alpha \cot (\beta)}_{I(\alpha)} \times\left[\begin{array}{ll}
0 & 1 \\
1 & 0
\end{array}\right] .
$$

The change of variables formula for quadratic forms implies that $\operatorname{det} M_{y}=(-2 i \alpha)^{2} \times$ $\operatorname{det} M_{w}$, where the first term on the right arises from the Wronskian of $\left\{Y_{0}^{+}, Y_{1}^{+}\right\}$. This implies that

$$
4 \cot (\beta)^{2}=4|A|^{2}|B|^{2}-\left(1-|A|^{2}-|B|^{2}\right)^{2} .
$$

From this, and lemma 4.1. one can numerically compute the phase angle $\beta$ and the integral $I$ as functions of $\alpha$. These are depicted in figure 2(b), In [3, Figure 1], these quantities were determined by numerically solving the initial-value problem 9 . The absolute and relative errors between the closed form solutions from equation (31) and the numerical approximations in [3] are depicted in figure 3 . This figure shows the approximations are extremely good, with a mean absolute error of approximately $\left.1.6 \times 10^{-9}\right]^{1}$ One can also show that $I\left(0^{+}\right)=-2 \cosh (\sqrt{7} \pi / 2) / \pi \cong-20.317$, which is in close agreement with figure $2(\mathrm{~b})$

\section{REFERENCES}

1. Milton Abramowitz and Irene A. Stegun (eds.), Handbook of mathematical functions with formulas, graphs, and mathematical tables, Dover Publications Inc., New York, 1992, Reprint of the 1972 edition. MR 1225604 (94b:00012)

2. Leo T. Butler, Invariant metrics on nilmanifolds with positive topological entropy, Geom. Dedicata 100 (2003), 173-185. MR 2011121 (2004j:37116)

\footnotetext{
${ }^{1}$ The angle $B$ reported in 3 Figure 1] equals $\pi+\beta$ in the present paper.
} 
FiguRE 3. Error in numerical integration of $I(\alpha)$ vs. closed form.

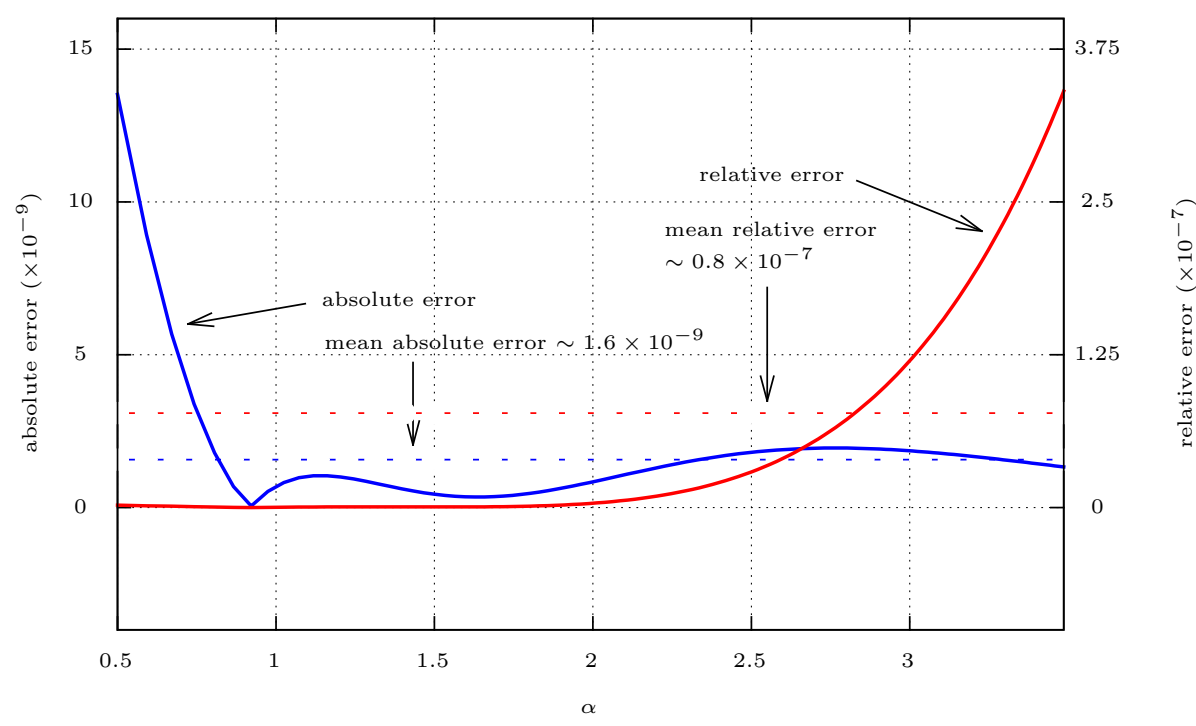

3. Leo T. Butler and Vassili Gelfreich, Positive-entropy geodesic flows on nilmanifolds, Nonlinearity 21 (2008), no. 7, 1423-1434, With online multimedia enhancements. MR 2425326 (2009g:37026)

4. M. W. Hirsch, C. C. Pugh, and M. Shub, Invariant manifolds, Lecture Notes in Mathematics, Vol. 583, Springer-Verlag, Berlin, 1977. MR 0501173 (58 \#18595)

5. Jerrold E. Marsden and Tudor S. Ratiu, Introduction to mechanics and symmetry, second ed., Texts in Applied Mathematics, vol. 17, Springer-Verlag, New York, 1999, A basic exposition of classical mechanical systems. MR 1723696 (2000i:70002)

6. R. Montgomery, M. Shapiro, and A. Stolin, A nonintegrable sub-Riemannian geodesic flow on a Carnot group, J. Dynam. Control Systems 3 (1997), no. 4, 519-530. MR 1481625 (98h:53069)

7. A. B. Olde Daalhuis, Hypergeometric function, NIST handbook of mathematical functions, U.S. Dept. Commerce, Washington, DC, 2010, pp. 383-401. MR 2655355

8. E. T. Whittaker and G. N. Watson, A course of modern analysis. An introduction to the general theory of infinite processes and of analytic functions: with an account of the principal transcendental functions, Fourth edition. Reprinted, Cambridge University Press, New York, 1962. MR 0178117 (31 \#2375)

9. S. L. Ziglin, Bifurcation of solutions and the nonexistence of first integrals in Hamiltonian mechanics. I, Funktsional. Anal. i Prilozhen. 16 (1982), no. 3, 30-41, 96. MR 674006 (85j:58066a)

10. Bifurcation of solutions and the nonexistence of first integrals in Hamiltonian mechanics. II, Funktsional. Anal. i Prilozhen. 17 (1983), no. 1, 8-23. MR 695092 (85j:58066b) 